\title{
Um comparativo entre métodos computacionais para planejamento de redes de telecomunicações
}

\author{
Rodrigo Marques de Figueiredo ${ }^{1}$ \\ José Vicente Canto dos Santos ${ }^{1}$
}

\begin{abstract}
Resumo: Com o crescimento da dependência da sociedade moderna de redes de telecomunicações confiáveis e eficientes, viu-se aumentada a necessidade de criação e planejamento de novas redes. $\mathrm{O}$ aumento do poder computacional, bem como o domínio de novas técnicas permitiu aos pesquisadores contar com novas ferramentas para auxiliarem no planejamento dessas redes, um problema em que a quantidade de soluções possíveis ultrapassa a capacidade humana de raciocínio. São avaliadas duas abordagens para sistemas de suporte ao planejamento de redes, qualitativa e quantitativa, para, dessa forma, indicar qual dessas possui um melhor desempenho. Será utilizada uma solução implementada a partir de algoritmos clássicos e outra a partir de um algoritmo genético. Um amplo estudo da bibliografia correlata foi feito para que o estudo de caso possa ser melhor avaliado. A comparação de desempenho é feita para dois cenários diferentes, um com menor e outro com maior número de nós. Após a aplicação das diferentes implementações computacionais, são apresentadas a avaliação individual e comparativa do desempenho das duas soluções.
\end{abstract}

Palavras-chave: Planejamento de redes. Redes de telecomunicações. Simulação.

\begin{abstract}
With the increasing dependence of modern society for reliable and efficient telecommunications networks, was created a huge need for creation and planning of these new networks. The increasing computing power and mastery of new computational techniques have enabled researchers in networks planning rely on these tools to assist the planning of these networks, a problem where the amount of possible solutions is beyond the ability of human reasoning. The analysis of two approaches for planning support systems of telecommunications networks will be qualitatively and quantitatively assessed in order to indicate which of the two approaches has a better performance. One of these solutions will be implemented from classic algorithms and the other from a genetic algorithm. An extensive review of the literature related was done to the case study can be better evaluated. The performance comparison is made for two different scenes, one smaller and one with a larger number of nodes. After the application of different computational implementations are carried out at individual and comparative assessment of the performance of the two solutions.
\end{abstract}

Keywords: Network planning. Simulation. Telecommunication networks.

\section{Introdução}

A sociedade moderna está tornando-se cada vez mais dependente de redes de telecomunicações eficientes e confiáveis, pois mais e mais produtos e serviços são lançados no mercado com a premissa de utilizar essas redes. Com a crescente demanda gerada por essas aplicações e até mesmo pelo número cada vez maior de pessoas com acesso a essas tecnologias, as empresas responsáveis por prover os serviços de telecomunicações, as operadoras, precisam investir na ampliação da infraestrutura. A ampliação da estrutura de telecomunicações é um problema importante de planejamento de redes, alvo de muitos estudos tanto por parte da academia como por parte da indústria.

\footnotetext{
${ }^{1}$ Programa de Pós-Graduação em Computação Aplicada, UNISINOS - São Leopoldo (RS) - Brasil \{marquesf, jvcanto@unisinos.br\}
}

http://dx.doi.org/10.5335/rbca.2013.2579 
Com o poder computacional atual e o conhecimento de técnicas computacionais de otimização de sistemas, os problemas de planejamento são modelados e avaliados por meio dessas ferramentas. O planejamento de redes de telecomunicações insere-se na gama de problemas complexos a serem solucionados por sistemas computacionais. Isso se explica pelo fato da relação do número de nós com as possibilidades de conexões criadas pela inclusão de cada nó na rede. Essa relação entre número de nós e possibilidade de enlaces é dada em uma relação fatorial, o que indica um sistema NP-Completo [1]. Com base nessa premissa, neste trabalho serão estudadas duas técnicas de suporte à decisão desenvolvidas para atender a necessidade de planejamento de redes, especialmente redes de telecomunicações de transporte.

A exploração do assunto por meio de um estudo das possíveis técnicas computacionais a serem aplicadas no planejamento das redes de telecomunicações é o objetivo deste trabalho, buscando focar nas técnicas mais indicadas para este tipo de problema. Portanto, será buscado traçar um comparativo entre duas técnicas de apoio a decisão, uma clássica e outra baseada em inteligência computacional. Dessa forma, haverá a possibilidade de traçar-se um comparativo entre as duas técnicas, levantando suas vantagens e desvantagens. Assim, este trabalho pode contribuir como fonte bibliográfica e técnica de avaliação para a definição de qual ferramenta computacional terá a melhor aplicabilidade a um dado planejamento de rede de telecomunicações de transporte.

Optou-se pela adoção de uma metodologia por meio de pesquisas em artigos científicos e livros, bem como pela avaliação quantitativa e qualitativa do desempenho das duas técnicas de apoio à decisão. Essa avaliação foi dada em um ambiente controlado, utilizando-se três diferentes cenários como fonte de dados a serem analisados.

\section{Métodos computacionais para solução do problema de planejamento em redes}

O estado da arte para soluções computacionais de problemas combinatórios, atualmente, é o uso de metaheurísticas, pois esta é a melhor maneira de ter-se uma resposta em tempo aceitável. Neste trabalho será realizado um comparativo entre uma solução obtida com algoritmos clássicos e outra solução original, que lança mão do uso de algoritmo genético para realizar o planejamento de uma rede de telecomunicações [2].

\subsection{Solução com algoritmos clássicos}

A abordagem clássica para o planejamento de redes é baseada em duas premissas básicas: roteamento pelo caminho mais curto e a determinação dos enlaces necessários dados pelo fluxo desses pela rede [3],[4]. O algoritmo de Dijkstra é o algoritmo que soluciona eficientemente o problema do caminho mais curto, chamado de PCMC. Já a determinação dos enlaces necessários à rede dada a demanda, fluxo de dados requisitado, é realizado por um algoritmo simples de busca geral em grafos. Este algoritmo resolve o problema da conexão simples [1].

\subsection{Solução com algoritmo genético}

Algoritmos genéticos são entes computacionais que utilizam métodos baseados na natureza, mais precisamente nos mecanismos de seleção natural [2]. John Holland foi o pioneiro nessa linha de trabalho com algoritmos genéticos, o objetivo de Holland era fundamentar a teoria geral de sistema de adaptação robusta [5].

O funcionamento de um algoritmo genético é baseado em um conjunto de soluções representado por cromossomos, chamado de população. Esta é avaliada em termos de seu desempenho, em um processo onde se dá a avaliação de sua adaptação, ou fitness. O resultado dessa avaliação indicará se a população estará apta a progredir ou se será dizimada. Estando apta a prosseguir, serão realizados cruzamentos e mutações, onde em cada um desses processos haverá sempre um passo intermediário que é o processo de verificação de fitness [6].

\section{Comparação das soluções para o problema de planejamento de redes de telecomunicações}

A metodologia utilizada para a comparação entre as soluções para o problema de planejamento de redes de telecomunicações é baseada na aplicação destas. Essa aplicação será dada na forma de estudo de caso, do qual farão parte dois cenários. Os cenários serão os mesmos que Canto dos Santos e Barreto [2] utilizaram em seu trabalho, no qual também foi utilizada a solução original através de algoritmo genético. 
O primeiro cenário foi retirado da biblioteca SNDlib [9], um repositório que contém instâncias de testes que são aplicados a problemas de otimização em redes de telecomunicações. A instância denominada de Di Yuan, formada inicialmente por 11 nós (vértices) e com demanda composta por 22 circuitos (arestas) será o primeiro cenário a ser testado. Nessa mesma biblioteca estão postas as demandas relativas a cada um dos circuitos.

Como segundo cenário foi escolhido uma rede de telecomunicações real, da antiga empresa Brasil Telecom ${ }^{\circledR}$ no estado do Rio Grande do Sul, cuja instância possui 27 nós (vértices) e 22 circuitos (arestas) com demanda. Esta instância foi retirada do trabalho de Piqueira [7]. É importante, em um trabalho no qual se propõe a avaliação de possíveis soluções para a indústria das telecomunicações, utilizar uma instância real, com suas restrições de demanda [8].

Ambas as soluções, com algoritmos clássicos e com algoritmo genético, foram implementadas para execução com o software MATLAB ${ }^{\circledR}$, que é amplamente aceito para aplicações computacionais como benchmark, tanto no âmbito acadêmico como no industrial. As soluções serão avaliadas em dois momentos distintos. Em um primeiro momento serão analisadas de maneira isolada e posteriormente serão comparadas. Acredita-se que dessa maneira ambas as soluções poderão ser avaliadas de maneira a determinar suas vantagens e desvantagens.

\subsection{Análise da solução através de algoritmos clássicos}

São basicamente dois os algoritmos que são utilizados em conjunto para que seja dada a solução para o problema de planejamento de uma rede de telecomunicações. Primeiramente a rede a ser planejada é submetida ao algoritmo de Dijkstra, o qual visa resolver o problema do caminho mais curto (PCMC). O resultado dado pelo algoritmo de Dijkstra será então submetido ao algoritmo que verifica as conexões necessárias para que as demandas sejam atendidas pela rede.

A premissa básica para que essa solução seja eficiente é a de que a rede possa assumir uma topologia inicial do tipo totalmente conectada, mais conhecida no meio como rede full matched. Essa premissa já mostra uma das desvantagens dessa solução, pois mesmo em se tratando de planejamento, algumas ligações podem ser inviáveis. Quaisquer que sejam os motivos que inviabilizem alguma ligação, isso não pode ser colocado facilmente no modelo como condição de contorno, portanto, esse fato deverá ser tratado no próximo passo da solução.

No passo de verificação das necessidades de conexão, as arestas com demandas são colocadas à disposição da rede resultante da etapa anterior. Com isso tem-se o planejamento da rede. Há outro ponto importante a ser realçado, que diz respeito à capacidade de avaliação da segurança da rede planejada. Como não são avaliadas possíveis falhas na rede, somente é avaliada a demanda requisitada de cada enlace, não existe a possibilidade de se prever algum enlace de proteção (backup). A seguir será possível verificar esses passos aplicados aos cenários de teste.

\subsubsection{Análise do cenário 1 - Rede de 11 nós}

A instância Di Yuan, retirada do repositório SNDlib, possui 11 vértices (nós) e 22 arestas (circuitos) com demandas. Esse é um repositório de referência para testar, avaliar e comparar modelos de projetos de rede e algoritmos, possibilitando o compartilhamento da informação, organizado por Pióro e Medhi [9]. Como mencionado anteriormente parte-se de uma rede totalmente conectada. Essa rede será analisada de maneira a verificar-se se existem conexões, circuitos que possam ser reaproveitados em termos de menor distância entre vértices. A maneira como o algoritmo de Dijkstra foi concebido prevê que seja avaliada a menor distância entre dois pontos, o que este resolve de maneira primorosa. Porém, quando se trabalha em um cenário de rede, existem outras situações que devem ser levadas em consideração para se ter um caminho ótimo. Em uma rede, o caminho ótimo pode não ser o mais curto, mas sim aquele que economiza mais recursos, no caso de redes, que minimize o número de enlaces.

Após rodar a implementação dos algoritmos clássicos sobre a rede de 11 nós, tem-se o resultado mostrado na Figura 1, na qual se pode notar que a resposta atende plenamente as necessidades requisitadas pelas restrições do problema, que são o menor caminho entre nós da rede e preservar os enlaces com demanda declarada. 


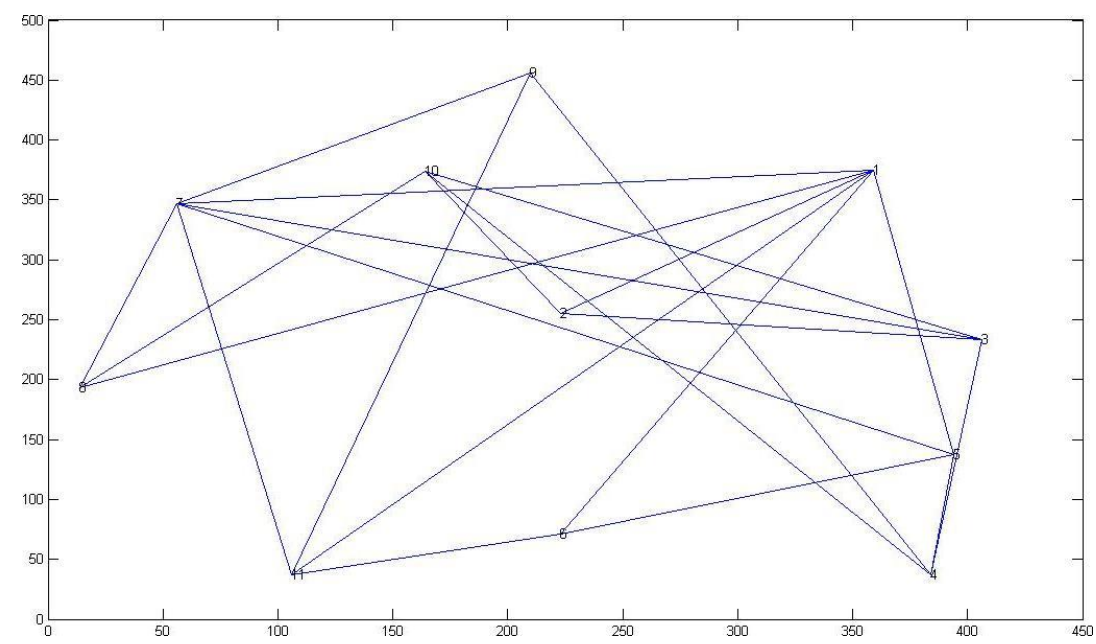

Figura 1: Solução com algoritmos clássicos para o planejamento da rede de 11 nós.

Atentando novamente para a Figura 1, nota-se que apesar de todas as demandas serem atendidas, o fator limitante para o número de circuitos, ou enlaces, é dado pelas próprias demandas. Em outras palavras, todos os enlaces com demandas são mantidos e os que não possuem são removidos. Isso ocorre em razão de que a menor distância entre cada um dos nós, quando comparados aos pares, é sempre a distância entre os próprios. Portanto, não há um ganho em termos de otimização, apenas garante-se que a rede atenderá as demandas.

\subsubsection{Análise do Cenário 2 - Rede de 27 nós}

Para o segundo cenário foi utilizada a rede com 27 nós e 45 circuitos, ou seja, uma rede com 27 equipamentos interligados por 45 enlaces. Essa é uma rede real, situada no estado do Rio Grande do Sul, como mencionado no início desta seção. A abordagem para essa instância de testes é a mesma utilizada para a anterior, parte-se de uma rede totalmente conectada como premissa básica. Esse procedimento será mantido para quaisquer que sejam os tamanhos das instâncias de teste.

Como se pode observar na Figura 2, o número de enlaces necessários para ligar totalmente a rede é extremamente elevado. Quanto mais vértices existirem na rede, maior será a necessidade de enlaces para torná-la totalmente conectada. Isso faz com que o tempo de processamento aumente de maneira diretamente proporcional ao número de nós que a rede possui.

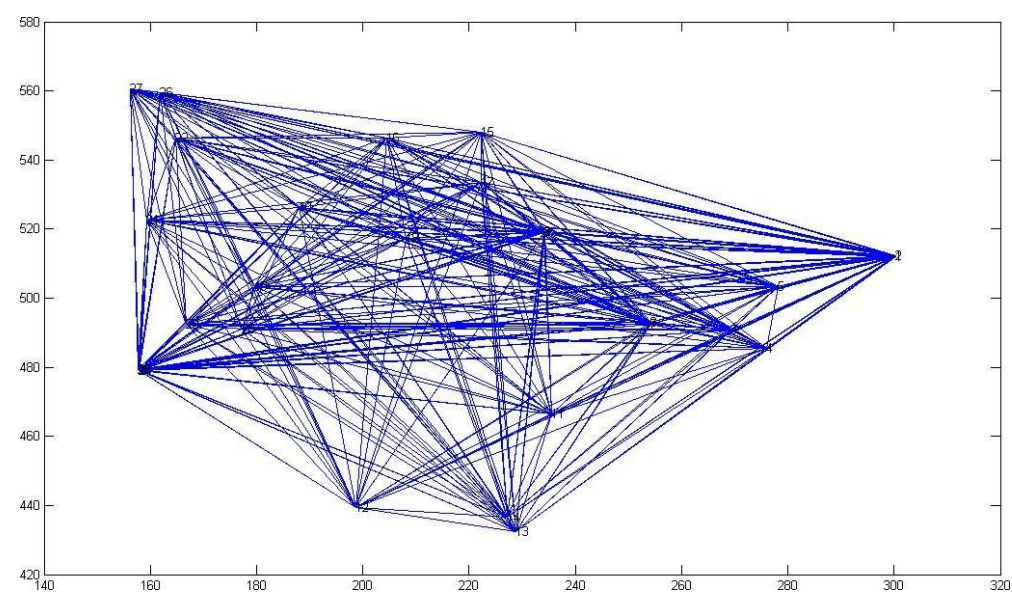

Figura 2: Rede de 27 nós, totalmente conectada.

Quando essa rede é submetida à implementação do sistema computacional baseado em algoritmos clássicos, esta passa a uma formatação topológica muito mais simples, Figura 3. Mas novamente pode-se notar 
que existe apenas uma redução baseada na manutenção dos enlaces que possuem demanda. Como existem 45 enlaces com demanda requerida, esses são mantidos e os demais excluídos da rede planejada.

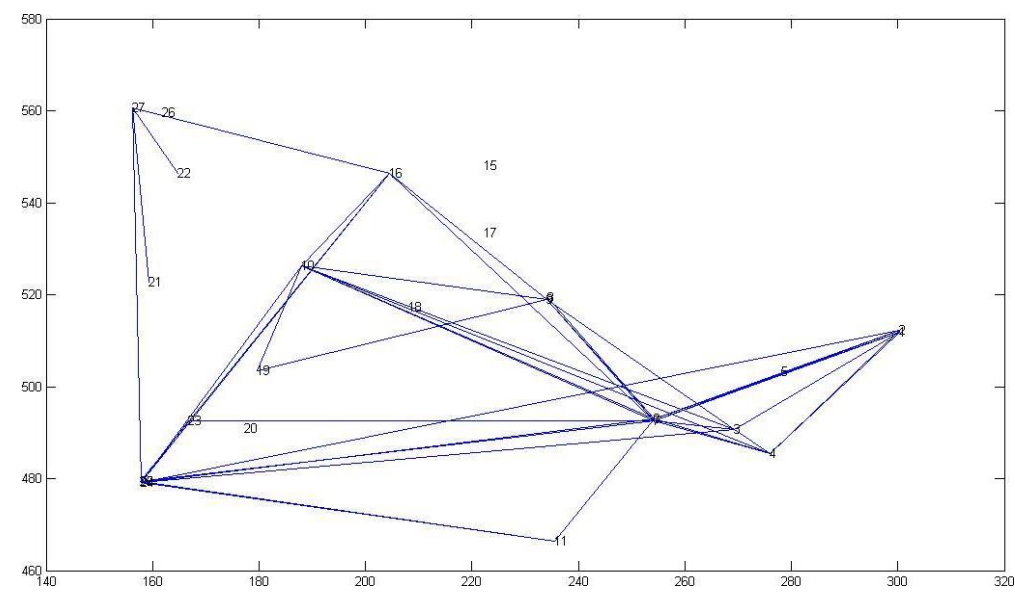

Figura 3: Solução com algoritmos clássicos para o planejamento da rede de 27 nós.

A Figura 3 é emblemática para mostrar como o planejamento de uma rede utilizando a abordagem clássica com um menor custo computacional é ineficiente. O nó 15 , por não possuir demanda, ficou fora da rede, já o nó 2 liga-se diretamente ao nó 24 , sem que perceba que poderia haver um reaproveitamento de enlace, ao passar pelo nó 5 e pelo nó 7. Com isso, pode-se observar que ao deixar o nó 15 fora da rede se estará também fragilizando-a como sistema, uma vez que em caso de surgimento de novas demandas, uma nova infraestrutura deverá ser construída. Essa prática não é interessante para as operadoras, pois implica a diminuição drástica da sua margem de lucro.

\subsection{Análise da solução através de algoritmo genético}

Para a solução original, que utiliza um algoritmo genético, é necessário se ter em mente que esta é uma solução baseada em uma meta-heurística. Assim, é uma solução na qual são utilizados conhecimentos prévios sobre o comportamento de redes de telecomunicações, para então estes fazerem parte do modelo de planejamento. Outro ponto importante a ser destacado é a natureza do algoritmo genético, que é um algoritmo evolutivo, totalmente dependente de iterações e avaliações do resultado atual para gerar um próximo.

Em uma solução baseada em algoritmo genético, como explicado anteriormente, existe uma função computacional de análise de desempenho da resposta, chamada de fitness, e que também pode ser chamada de Função Objetivo (F. O.). Na implementação realizada em Canto dos Santos e Barreto [2], basicamente existem três quesitos de avaliação que formam a F.O. do sistema:

1. Custo da topologia: avalia a distância total de enlaces realizados entre cada nó;

2. Custo de roteamento: avalia o atendimento das demandas considerando a topologia proposta;

3. Custo do tráfego pela distância: avalia a relação de distância por tráfego demandado.

Quando a implementação baseada em algoritmo genético inicia é realizada uma proposta de solução, avaliada quanto à sua capacidade de atender a função objetivo. Caso a F.O. seja atendida, essa solução é mantida como semente para a próxima, a nova proposta parte desta. Caso contrário, no entanto, esta resposta é eliminada e uma nova é dada. Isto ocorre de maneira iterativa até que o algoritmo chegue a seu fim. $\mathrm{O}$ fim do algoritmo pode ser determinado de duas formas: a primeira é o alcance de um número pré-determinado de iterações, a segunda é testar a qualidade da resposta e verificar a F.O. quanto a um valor também pré-determinado.

Durante as mutações, as propostas de soluções aleatórias são testadas de maneira a avaliar se alguma característica da rede poderia ter ficado de fora das propostas anteriores. Cada uma das propostas é uma topologia, portanto, tanto as avaliações como as mutações são dadas diretamente na topologia. Com isso, se pode afirmar que a topologia proposta vai evoluindo, até chegar a um ponto no qual é aceita ou tem seu ciclo interrompido por alcançar o número máximo de iterações. 
Cada iteração cria uma geração candidata à resposta do problema de planejamento da rede, portanto, a primeira geração tem uma qualidade de resposta inferior à da sua sucessora e assim por diante. Canto dos Santos e Barreto [2] determinaram em seu trabalho através de sucessivos testes com diversos benchmarks de redes que 100 gerações é o suficiente para que se tenha uma boa aproximação de um planejamento ótimo para a rede. Será analisado agora o comportamento da implementação com algoritmo genético frente aos dois cenários considerados neste trabalho.

\subsubsection{Análise do cenário 1 - rede de 11 nós}

Diferentemente da solução por algoritmos clássicos para esta abordagem, não há nenhuma premissa que deva ser respeitada para que se possa iniciar o procedimento, pois o algoritmo genético propõe a resposta e o próprio avalia se esta atende ou não os requisitos mínimos para ser aceita. Foram realizadas 100 iterações, de acordo com os experimentos apresentados em Canto dos Santos e Barreto [2], portanto tem-se 100 respostas para ser a topologia da rede planejada.

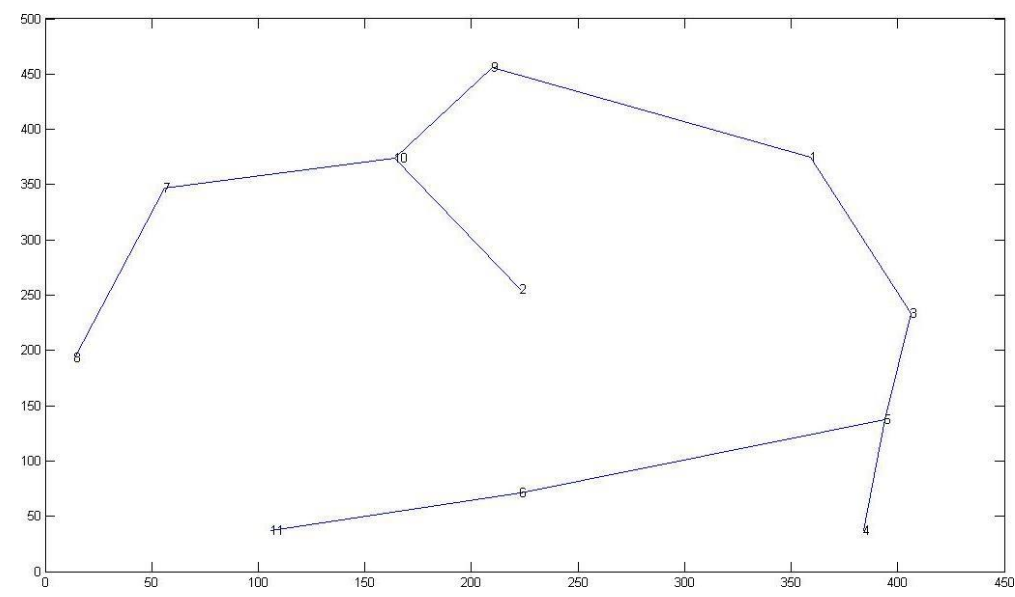

Figura 4: Solução para rede de 11 nós com implementação por algoritmo genético.

A Figura 4 mostra a resposta correspondente à centésima geração. Nessa topologia, a rede tem todas as suas demandas atendidas e são respeitados os menores caminhos entre nós - levando-se em consideração toda a rede -, bem como o menor custo de distância por demanda. Percebe-se, então, que é uma solução muito próxima da ótima, pois todas as demandas são atendidas com um baixo custo de infraestrutura, pela minimização do número de enlaces. 

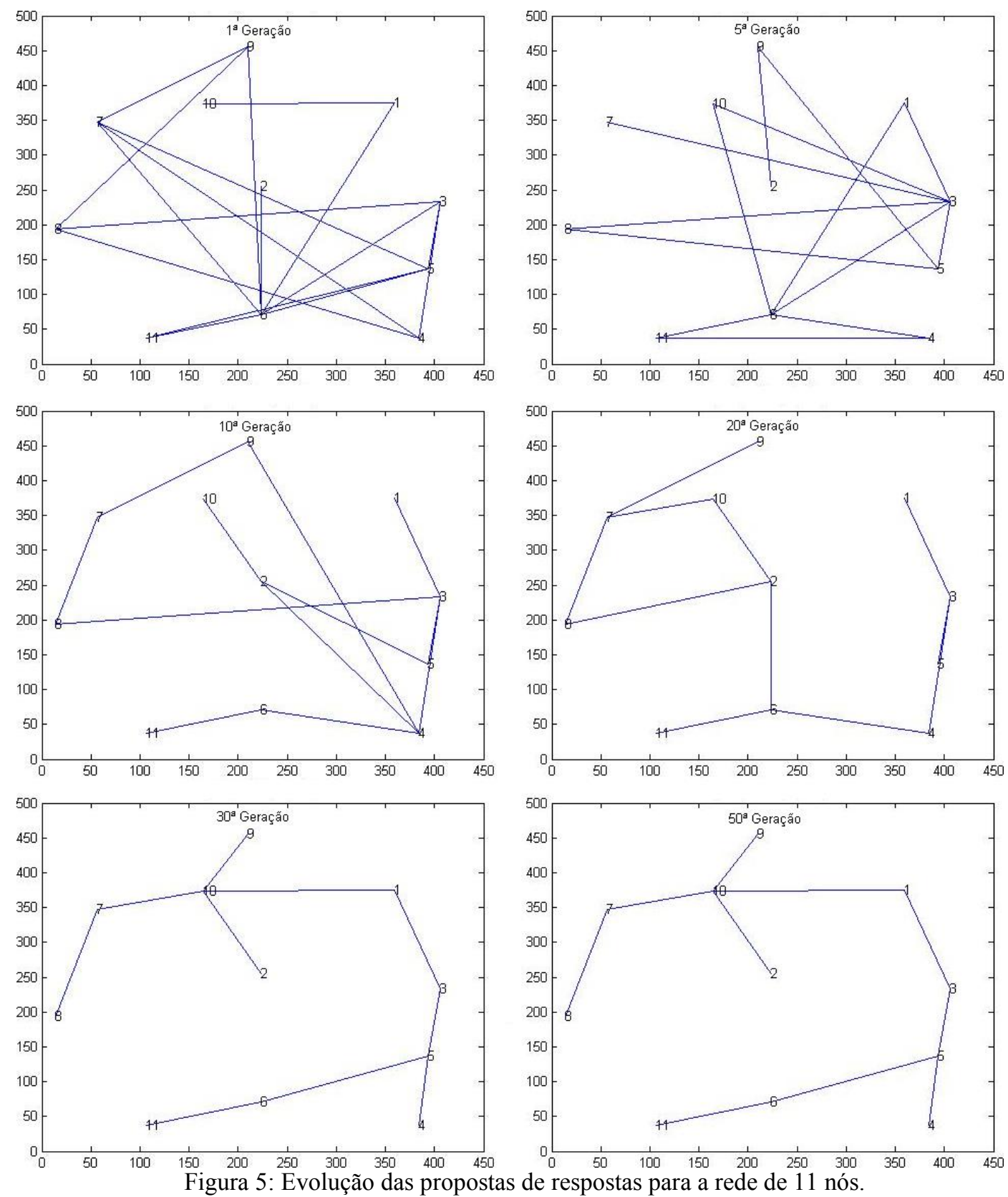

A evolução pela qual as topologias propostas para o planejamento dessa rede de telecomunicações passaram pode ser verificada na Figura 5. O grafo do canto superior esquerdo é correspondente à primeira geração, com relação ao qual já se pode notar uma evolução para a proposta da quinta geração, representado pelo grafo do canto superior direito. Os últimos grafos mostram a trigésima e a quinquagésima gerações na porção inferior da Figura 5, onde se observa uma resposta significativamente mais otimizada, evoluída. Outro ponto importante a se observar é que da trigésima para a quinquagésima geração não houve mudança na topologia proposta, porém, mais adiante, houve alguma mutação, que fez com que a resposta final fosse a dada pela centésima geração, mostrada na Figura 4.

\subsubsection{Análise do Cenário 2 - Rede de 27 nós}

Com a análise da solução dada pela implementação feita com algoritmo genético realizada sobre o cenário 2 , pode-se ter uma visão melhor da performance dessa proposta. Devido às dimensões desse cenário real, estressa-se o sistema computacional com maior intensidade do que no cenário anterior. 
Tem-se na Figura 6 o resultado dado pela centésima geração do algoritmo genético. Pode-se observar que com o aumento da rede planejada a simplificação foi menor. Houve certamente um maior esforço do algoritmo, principalmente na avaliação dos desempenhos de cada geração (topologia) de maneira a atribuir a essas gerações maior valor. Um ponto importante a ressaltar é a ligação entre os vértices 22,26 e 27 , em que não existe uma conexão entre os nós 26 e 27, que poderia simplificar um dos enlaces desses com o nó 22 . Isso ocorre em razão de que não existe demanda entre 26 e 27 e não houve nenhuma mutação que forçasse tal conexão. Portanto, aqui existe um dos pontos fracos do algoritmo. Quando não há uma restrição que force um enlace, este só será sugerido caso haja uma mutação na próxima geração, processo que ocorre de maneira aleatória.

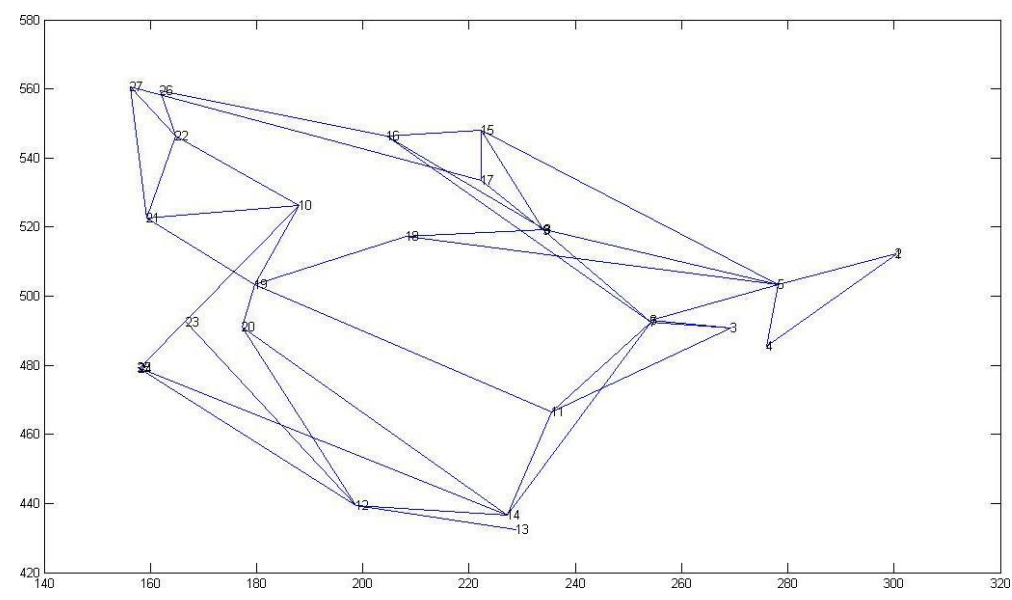

Figura 6: Solução do sistema computacional baseado em algoritmo genético para rede de 27 nós.

Ainda sobre a Figura 6, percebe-se que o fato que ocorre entre os nós 22, 26 e 27, anteriormente descrito, acontece em mais regiões do grafo, como por exemplo entre os nós 15,16 e 17. Outra característica trazida pelo algoritmo genético para esse grafo está na região dos pontos 12, 13 e 14, onde, por distância, claramente haveria um enlace entre 13 e 14, mas como esta não possui uma demanda declarada a F.O. não consegue perceber. Apesar desses pontos ressaltados revelarem uma certa fragilidade na implementação via algoritmo genético, esta ainda prova ser interessante, pois consegue de maneira inteligente gerar propostas evolutivas para o planejamento de uma rede de telecomunicações.

Essa evolução pode ser vista na Figura 7, onde estão dispostas as topologias de alguns pontos do processo evolutivo do algoritmo genético. Assim como na Figura 5, no canto superior esquerdo está colocada a primeira geração posta pelo algoritmo genético. Ao seu lado está a quinta geração, sendo possível perceber que nessas existe pouca diferença de uma rede totalmente conectada. Mas a evolução dada a partir da vigésima geração é notória, como pode ser confirmada na quinquagésima geração, apresentada no canto inferior direito da Figura 7. Dessa forma, tem-se, agora, informações suficientes para fazer um comparativo direto entre as duas soluções, analisando a solução final para cada cenário de teste. 

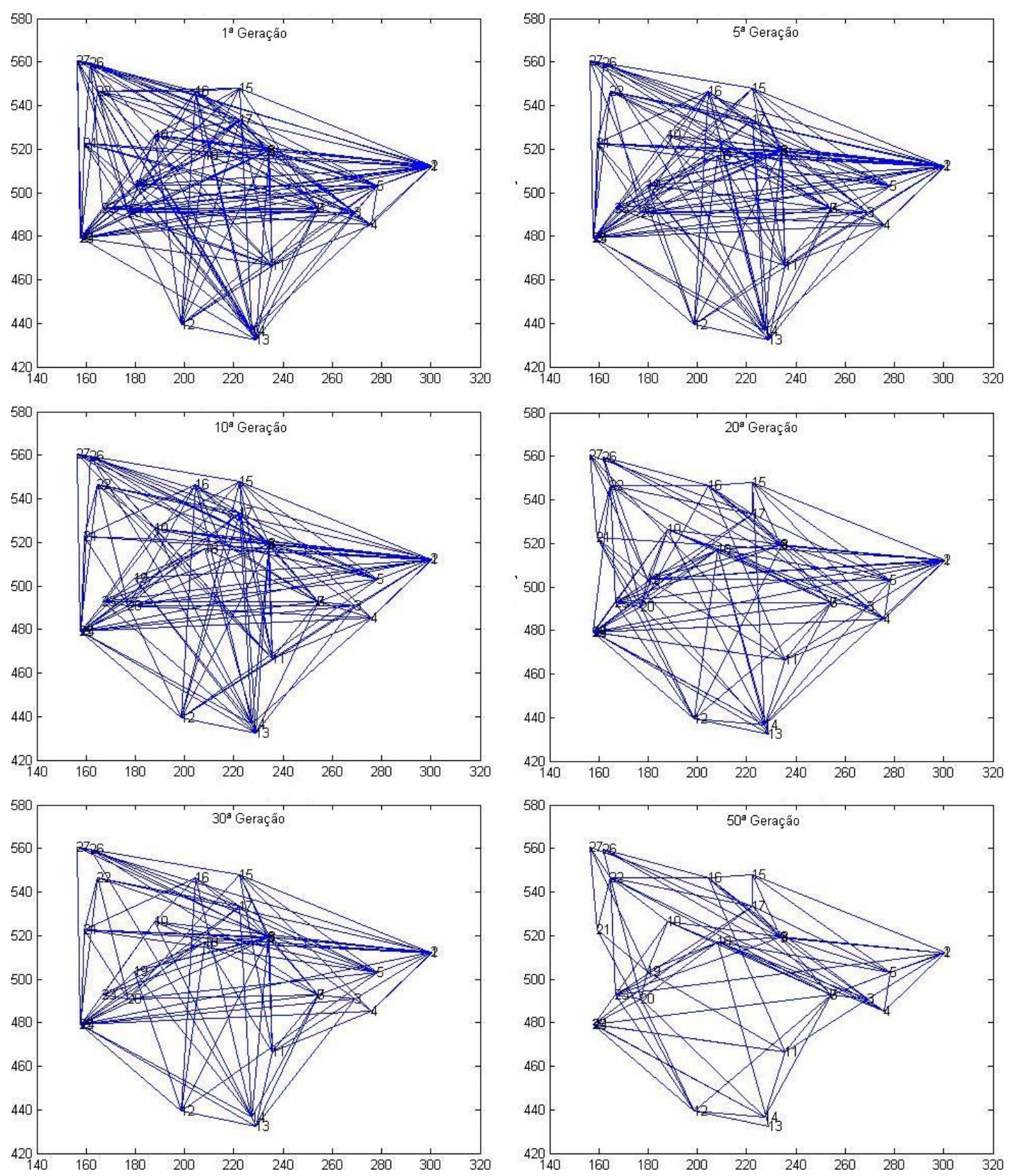

Figura 7: Evolução das propostas de respostas para a rede de 27 nós.

\subsection{Comparação entre as implementações}

O principal objetivo deste trabalho é traçar um comparativo entre as duas soluções estudadas, a solução implementada com algoritmo clássico e a implementada por meio de algoritmo genético. Manter os testes em cenários conhecidos e controlados é de suma importância para que a análise possa ser feita da melhor maneira. A análise comparativa ocorrerá em duas etapas, iniciando pela comparação de cada cenário e finalizando com uma análise geral de ambas as implementações.

\subsubsection{Comparação entre as implementações no cenário 1}

No cenário 1 existem 11 nós (equipamentos) com 22 enlaces (circuitos) com demandas declaradas e todos os nós (vértices) possuem contribuição para estas. As restrições postas fazem com que haja a necessidade de 
atender todo esse fluxo de demanda através da rede com a visita de todos os nós. Essa premissa é confirmada na solução dada pela implementação com algoritmo clássico, como pode ser visto na Figura 1 e também na solução da implementação com algoritmo genético, mostrada na Figura 4.

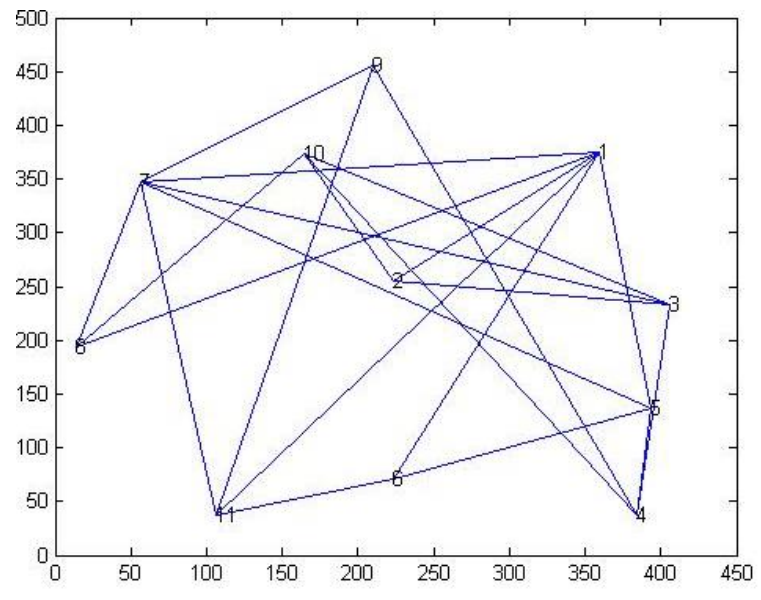

(a)

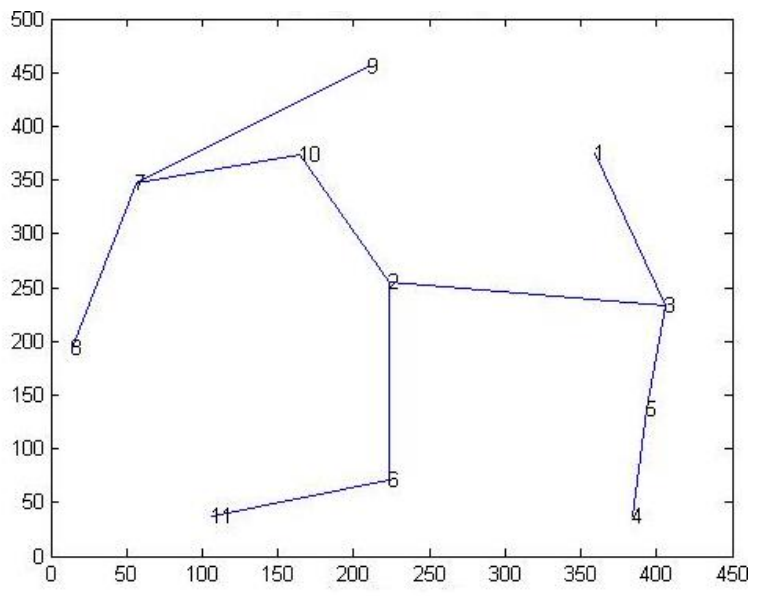

(b)

Figura 8: Comparativo das soluções para o cenário 1.

Os resultados colocados lado a lado na Figura 8 facilitam sobremaneira a comparação entre as duas implementações. Observando a solução dada pela implementação com algoritmos clássicos, na Figura 8a, percebe-se um claro excesso de enlaces, sendo a demanda distribuída por enlaces individuais que atendem cada par de vértices. Já a Figura $8 \mathrm{~b}$ mostra uma rede plenamente otimizada, onde todos os nós (vértices) são visitados por um número pequeno de enlaces, na verdade um número mínimo destes.

Existe uma clara vantagem, qualitativamente visível, da solução dada com a implementação com algoritmo genético sobre a implementação clássica. Na nova abordagem a garantia de um menor custo por distância juntamente com a demanda, cria um cenário com menos enlaces necessários para o atendimento das restrições do problema de planejamento da rede de telecomunicações. De maneira quantitativa, observou-se que a implementação baseada em algoritmos genéticos chegou a solução em 80,35 segundos, enquanto a baseada em algoritmos clássicos levou 292,67 segundos. Nota-se, assim, a eficiência do algoritmo genético para esse tipo de problema.

\subsubsection{Comparação entre as implementações no cenário 2}

Aplicando os sistemas computacionais baseados em algoritmos clássicos e também o baseado em algoritmo genético, pode-se avaliar as performances, confrontando-as. As informações visuais presentes nas Figuras 3 e 5 foram compiladas na Figura 9 para que possam ser observadas com melhor facilidade. Nela podese observar a principal diferença entre a topologia proposta pela implementação com algoritmos clássicos em relação à realizada com algoritmo genético, que é a conectividade da rede. 


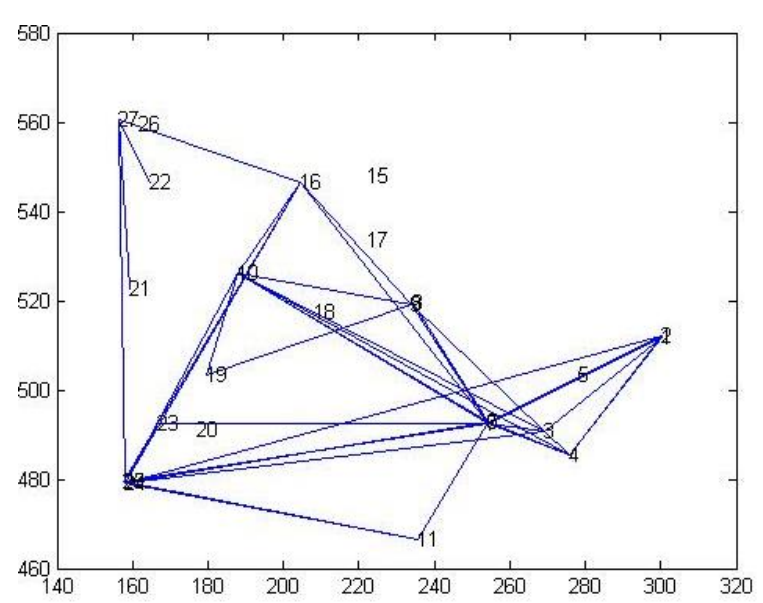

(a)

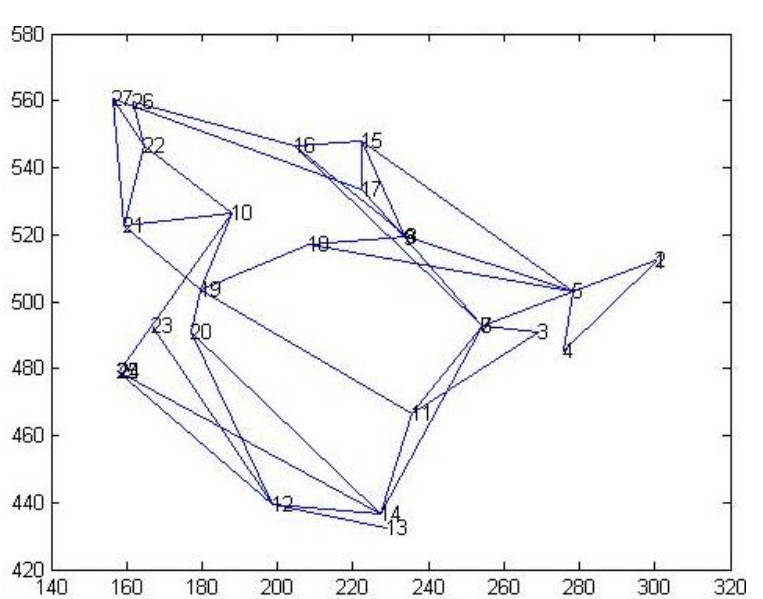

(b)

Figura 9: Comparativo das soluções para o cenário 2.

Esta avaliação em termos de conectividade da rede é uma análise qualitativa. Na Figura 9a tem-se a resposta proposta pelo sistema baseado em algoritmos clássicos, onde se nota claramente que os nós ficam fora da topologia proposta. Em uma rápida consulta à base de dados confirma-se que isso ocorre, pois não há demanda requisitada por esses nós. Já na proposta de solução dada pela implementação com algoritmos genéticos, existe uma conectividade total entre os nós, pois todos são visitados por enlaces, independentemente de formarem ou não ciclos. De maneira quantitativa, observou-se que os tempos demandados para solução são bastante diferentes. O algoritmo genético levou 165,71 segundos para obter a solução, tempo muito inferior aos 792,67 segundos gastos pelo algoritmo clássico para chegar à sua resposta.

Sem dificultar de maneira drástica a implementação, o sistema computacional baseado em algoritmos clássicos não será capaz de garantir a conectividade, pois para isto este terá que prever a avaliação do custo por conexão, o que garante a melhor conectividade [8]. No sistema computacional baseado em algoritmos genéticos, essa avaliação faz parte da função objetivo que avalia o desempenho das topologias propostas. Assim sendo, a conectividade sempre tenderá a ser atendida, desde que o sistema possa evoluir, conforme mostram as Figuras 4 e 6. Uma rede que tenha sua conexão atendida de maneira otimizada terá, por consequência, uma robustez proporcional, pois na falha de algum equipamento (o nó deixa de existir) haverá a possibilidade de outros enlaces fornecerem o caminho para que as demandas que por este passavam sejam atendidas. Portanto, a solução dada pelo sistema baseado em algoritmo genético possui uma melhor qualidade em relação à solução baseada em algoritmos clássicos.

\section{Conclusão}

O principal objetivo deste trabalho, a avaliação de duas soluções computacionais de apoio ao planejamento de redes de telecomunicações, foi plenamente alcançado. Para os testes foi utilizada uma solução baseada em algoritmos clássicos, desenvolvida pelos autores do presente trabalho e outra solução baseada em algoritmo genético, desenvolvida em Canto dos Santos e Barreto [2]. Ambas implementadas em MATLAB ${ }^{\circledR}$, para poderem ser avaliadas em um mesmo ambiente.

Em um comparativo direto com a implementação por meio dos algoritmos clássicos, observa-se que o uso dos algoritmos clássicos para o planejamento, sem que haja uma modelagem específica, que leve em conta restrições de vértices já visitados, por exemplo, acaba sendo ineficiente. Porém, quando o número de vértices aumenta, pode ocorrer uma explosão no tempo computacional, podendo inviabilizar o uso da técnica. Este tipo de solução é interessante para redes de um certo tamanho, onde estas sejam pequenas o suficiente para que o custo de enlaces extras seja minimizado, e que não haja uma explosão computacional.

Considerando as análises realizadas, notou-se uma clara vantagem da solução apresentada em Canto dos Santos e Barreto [2], a qual utiliza como base um algoritmo genético, frente à solução dada por algoritmos clássicos. Nessa análise qualitativa, sob um olhar crítico e com conhecimento específico, nota-se que a solução via algoritmos genéticos é mais robusta. O principal motivo para isso é a filosofia dos algoritmos genéticos e 
implementação realizada em Canto dos Santos e Barreto [2]. Foi passado ao algoritmo genético o objetivo de atender as premissas, ou requisitos, apresentados na seção 3.2, onde uma Função Objetivo (F.O.) foi definida para determinar, dessa forma, a evolução das respostas do sistema.

O desenvolvimento deste trabalho foi estimulante e deixou portas abertas para trabalhos futuros, em duas frentes. A primeira é melhorar o modelo do sistema baseado em algoritmos clássicos, buscando mimetizar a inteligência presente no algoritmo genético via a criação de um modelo matemático maior e mais representativo. Dessa forma, se poderia avaliar quantitativamente, em termos de tempo computacional, as duas soluções, uma vez que qualitativamente elas seriam equivalentes, partindo da premissa que este trabalho comprovou que existe uma diferença qualitativa entre as duas implementações. A outra frente de trabalho seria a melhoria do sistema baseado em algoritmo genético desenvolvido em Canto dos Santos e Barreto [2], no qual poderiam ser colocados novos parâmetros na função objetivo com o intuito de refinar e aprimorar ainda mais a resposta do sistema quanto à conectividade e segurança, bem como restrições físicas da rede, como capacidades de equipamentos.

\section{Referências}

[1] GOldBARG, M., CESAR, L., PACCA, H. Otimização Combinatória e Programação Linear: Modelos e Algoritmos. Rio de Janeiro: Campus 2000.

[2] CANTO DOS SANTOS, J. V.; BARRETO, M. B. Computational System Based on Genetic Algorithm for Planning and Restoration of Telecommunication Networks. In: IADIS International Conference APPLIED COMPUTING 2011, Rio de Janeiro - RJ. Proceedings of the IADIS International Conference APPLIED COMPUTING 2011, v. Único. p. 241-248.

[3] COHEN, E. Approximate Max Flow on Small Depth Networks. AT\&T-Bell Laboratories. Murray Hill, 1974.

[4] CAO, Z., FAN, P., WANG, H. Analysis of Maximum Flow in Random Graphs for Network Coding. IEEE International Symposium on Microwave, Antenna, Propagation and EMC Technologes for Wireless Communications Proceedings, 2005.

[5] HILLIER, F. S., LIEBERMAN, G. J.. Introdution to Operations Research. 8th Edition, New York: McGraw-Hill, 2005.

[6] BISCHOFF, E. Estudo da utilização de Algoritmos Genéticos para Seleção de Redes de Acesso. 2009. Dissertação (Mestrado em Engenharia Elétrica), Publicação PPGENE.DM-372/09 - Departamento de Engenharia Elétrica, Universidade de Brasília, Brasília.

[7] PIQUEIRA, A. Metodologia para evolução topológica de redes ópticas de transmissão - Implantação da Tecnologia ASON. 2008. Dissertação (Mestrado em Engenharia Elétrica), Publicação PPGENE.DM - 059/08 - Departamento de Engenharia Elétrica, Universidade de Brasília.

[8] WHITE, A, R, P., MANN, J, W., SMITH, G, D. Genetic Algorithms and Network Ring Design. Annals of Operations Research v. 86. p 347 - 371, 1999.

[9] PIÓRO, M., MEDHI, D.; Routing, Flow, and Capacity Design in Communication and Computer Networks. $1^{\text {st }}$ ed. Elsevier, 2004. 